\title{
Uso da Série Ondaleta Bidimensional Chapéu Mexicano na Parametrização de Campos de Velocidades Sísmicas
}

\author{
Marcus V. T. Mello*, UFBA, Salvador, BA, Brasil \\ Wilson M. Figueiró, CPGG-IGEO-UFBA, Salvador, BA, Brasil
}

Copyright 2014, SBGf - Sociedade Brasileira de Geofísica.

Este texto foi preparado para a apresentação no VI Simpósio Brasileiro de Geofísica, Porto Alegre, 14 a 16 de outubro de 2014. Seu conteúdo foi revisado pelo Comitê Técnico do VI SimBGf, mas não necessariamente representa a opinião da SBGf ou de seus associados. É proibida a reprodução total ou parcial deste material para propósitos comerciais sem prévia autorização da SBGf

\begin{abstract}
Geological models are theoretical qualitative objects of high complexity. It is desirable and useful to obtain a quantitative representation of its physical aspects (compressional seismic velocity field, in the case of this work), by means of parameterization. It becomes possible the mathematical manipulation of such models. This work shows an application of wavelet series for such purpose. The wavelet series is a tool already in use by allowing the representation of complicated functions as linear combinations of simpler ones. Specifically, a two-dimensional Mexican Hat wavelet series is used to represent (parameterize) models of two-dimensional seismic velocity field.
\end{abstract}

Keywords: Mexican hat wavelet, Parameterization, and two-dimensional seismic velocity field.

\section{Resumo}

Modelos geológicos são objetos teóricos de alta complexidade e de caráter qualitativo. Obter uma representação quantitativa destes modelos, a chamada parametrização, mesmo que apenas de alguns de seus aspectos (como a velocidade sísmica, foco deste trabalho), é desejável e útil, pois os tornam mais simples e passíveis de manipulação matemática. Este trabalho mostrará uma aplicação da série ondaleta para este propósito. A série ondaleta é uma ferramenta já consagrada por possibilitar a representação de funções complicadas como combinações lineares de outras mais simples. Especificamente, será usada a série bidimensional (pois há a concepção do modelo bidimensional para as velocidades sísmicas), tendo como base a ondaleta do tipo chapéu mexicano.

Palavras chaves: Ondaleta Chapéu Mexicano, Parametrização e campo de velocidades sísmicas bidimensionais.

\section{Introducão}

As ondaletas são funções matemáticas capazes de decompor, representar ou descrever outras funções, de forma a permitir a análise destas em diferentes escalas. Em certo sentido, a análise de uma função por série ondaleta guarda alguma semelhança com a análise em série de Fourier. Por outro lado, mostra-se muito mais eficaz na análise de sinais não estacionários. A aplicação desta ferramenta é vasta: devido à sua capacidade em decompor funções de complexidade elevada, segundo uma base (composta por outras funções mais simples) proveniente da ondaleta considerada. As ondaletas são ferramentas poderosas no processamento de sinais. Além disso, são muito bem aplicadas na: compressão de dados, meteorologia, economia e estatística (Morettin, 1999).

Neste trabalho será mostrada uma aplicação das séries ondaletas a fim de se obter uma eficaz parametrização de um dos aspectos de um modelo geológico: a velocidade das ondas sísmicas compressionais em subsuperfície.

A ideia é que, em um certo modelo geológico, para cada ponto $(x, z)$ existe uma $v(x, z)$ que é a velocidade da onda sísmica no referido ponto. Admite-se que sejam conhecidos $x, z$ e $v(x, z)$, como uma distribuição discreta de valores, mas não a sua expressão matemática contínua. Construir tal expressão, através da análise por série ondaleta bidimensional, é o objetivo deste trabalho. Outros trabalhos, em geofísica, possuem abordagens parecidas (por exemplo: há trabalhos que se valem das ondaletas do tipo Haar e outros do tipo Morlet), nestes, os campos de velocidades são parametrizados por séries ondaletas unidimensionais (Martinez \& Figueiró, 2011; Perin \& Figueiró, 2011; e Bastos \& Figueiró, 2013). O presente trabalho propõe desdobramentos ainda não considerados pelos trabalhos mencionados, tais como:

1. O tipo de ondaleta usada, que será a chapéu mexicano; e

\section{A implementação da série ondaleta bidimensional.}

\section{Aspectos Teóricos}

As ondaletas podem ser pensadas como alternativas a outros sistemas de funções, usados como bases para representação de funções (Morettin, 1999) pertencentes a certos espaços (como senos e cossenos, polinômios ortogonais, funções de Haar, Walsh, etc).

Bases de Fourier (cuja composição é de funções trigonométricas) são localizadas na frequência, mas não no tempo; já as ondaletas localizam-se no tempo (ou no espaço) e na frequência, o que as torna ideais para analisar sinais não estacionários. A filosofia, tanto na análise usando a série ondaleta, quanto a de Fourier (ou qualquer outra base) é aproximar uma função por combinações lineares de funções que constituem a respectiva base. Comparativamente, são necessárias menos ondaletas do que senos e cossenos (como no caso da análise de Fourier) para aproximar funções com picos e 
descontinuidades, obtendo assim, via de regra, resultados mais acurados com menor custo computacional.

Funções periódicas, cujos períodos sejam $2 \pi$ e quadrado integráveis, $L^{2}(0,2 \pi)$, são geradas (na análise de Fourier) por superposições de exponenciais complexas, $\phi_{n}(x)=$ $e^{i n x}, n=0, \pm 1, \pm 2, \ldots$, obtidas por dilatações da função $\phi(x)=e^{i x}$ ou seja, $\phi_{n}(x)=\phi(n x)$. A ideia é aplicar este raciocínio para todo o $L^{2}(\mathbb{R})$, quer dizer, gerar este espaço partindo de uma única função, $\psi$. Isso pode ser conseguido através de translações e compressões (ou dilatações) de $\psi$ :

$$
\psi_{j, k}(t)=2^{j / 2} \psi\left(2^{j} t-k\right) ; j, k \in \mathbb{Z}
$$

A ondaleta (função $\psi$ ) usada neste trabalho é do tipo Chapéu Mexicano, obtida pela segunda derivada da função densidade de probabilidade gaussiana ${ }^{1}$. É contínua (diferentemente da Haar), suave, simétrica em relação ao eixo vertical e tende assintoticamente para zero quando $|t| \rightarrow \infty$. Sua expressão matemática é:

$$
\psi(t)=\frac{2}{\pi^{1 / 4} \sqrt{3 \sigma}}\left(1-\frac{t^{2}}{\sigma^{2}}\right) e^{-\frac{t^{2}}{2 \sigma^{2}}}
$$

A base do sistema que será usado para formar funções dá-se pela combinação de (1) com (2), resultando em:

$$
\psi_{j, k}(t)=2^{j / 2} \frac{2}{\pi^{1 / 4} \sqrt{3 \sigma}}\left(1-\frac{\left(2^{j} t-k\right)^{2}}{\sigma^{2}}\right) e^{-\frac{\left(2^{j} t-k\right)^{2}}{2 \sigma^{2}}}
$$

Retomando a ideia principal, objetiva-se escrever uma função bidimensional qualquer $f(x, y) \in \mathbb{R}^{2}$ como combinação linear das ondaletas que formam a base gerada por (3). Pode-se escrever esta combinação por meio de somatórios, obtendo a função $f(x, y)$ como segue:

$$
f(x, y)=\sum_{\mathbf{I}} d_{\mathbf{I}} \mu_{\mathbf{I}}(x, y),
$$

em que I é um vetor de índices,

$$
\mathbf{I}=\left(j_{1}, j_{2}, k_{1}, k_{2}\right)
$$

e a ondaleta bidimensional $\mu_{\mathbf{I}}(x, y)$ é dada por:

$$
\mu_{\mathbf{I}}(x, y)=\psi_{j_{1}, k_{1}}(x) \cdot \psi_{j_{2}, k_{2}}(y) .
$$

Os coeficientes $d_{\mathbf{l}}$ que aparecem em (4) podem ser obtidos do seguinte modo:

$$
d_{\mathbf{I}}=\left\langle f, \mu_{\mathbf{I}}\right\rangle=\iint_{\mathbb{R}^{2}} f(x, y) \mu_{\mathbf{I}}(x, y) \mathrm{d} x \mathrm{~d} y .
$$

\footnotetext{
${ }^{1}$ Em teoria estatística, a função densidade de probabilidade gaussiana contém dois parâmetros, $\mu$ e $\sigma$. O primeiro, $\mu$, representa a média dos valores (aqui $\mu=0$, resultando que $t=0$ torna-se o eixo de simetria do gráfico da função); já o segundo parâmetro, $\sigma$, simboliza o desvio padrão dos dados. Neste trabalho, entretanto, o valor de $\sigma$ foi arbitrado em 1,22 (este valor originou melhores resultados; note-se que $\sigma=1$, levaria à função chapéu mexicano que aparece comumente em outros textos como em (Morettin, 1999)
}

Entretanto, este processo deve ser aplicado tão somente quando a função ondaleta escolhida forma uma base ortonormal, mesmo para ondaletas bidimensionais. No caso da Chapéu Mexicano, utilizada neste trabalho, a base formada não é ortonormal. Os coeficientes $d_{\mathbf{l}}$ serão calculados com a técnica dos mínimos quadrados:

$$
d_{\mathbf{I}}=\left(\underline{\mu}^{T} \underline{\mu}\right)^{-1} \underline{\mu}^{T} \underline{f}
$$

\section{Metodologia}

Com base nas equações (4) e (6) o objetivo é obter uma função $v(x, z)$ que descreva a velocidade sísmica no modelo como função da distância $x$ e da profundidade $z$ :

$$
v(x, z)=\sum_{\mathbf{I}} d_{\mathbf{I}} \mu_{\mathbf{I}}(x, z),
$$

em que

$$
\mu_{\mathbf{I}}(x, z)=\psi_{j_{1}, k_{1}}(x) \psi_{j_{2}, k_{2}}(z)
$$

A equação (4) - e consequentemente a (9) - são verdadeiras para somatório infinito ou seja, para os valores dos índices $j_{1}, j_{2}, k_{1}, k_{2}$ variando de $-\infty$ à $\infty$. Na prática, uma escolha de valores convenientes para os índices torna a quantidade de coeficientes $d_{\boldsymbol{l}}$ menor e garante, com boa aproximação, que estas equações ainda serão válidas. O processo de escolha destes índices, neste trabalho, foi empírica; a experiência tem mostrado que os melhores resultados são obtidos com valores simétricos para os índices $j$. Já para os índices $k$, os valores parecem se relacionar com as dimensões do modelo; por exemplo, para um modelo de $9 \mathrm{~km}$ de extensão e $3 \mathrm{~km}$ de profundidade, os valores poderiam ser

$$
\begin{array}{cccc}
j_{1 m}=-3 & j_{1 M}=3 & j_{2 m}=-3 & j_{2 M}=-3 \\
k_{1 m}=0 & k_{1 M}=9 & k_{2 m}=0 & k_{2 M}=3
\end{array}
$$

As notações $\mathrm{m}$ e $\mathrm{M}$ simbolizam respectivamente os valores mínimos e máximos de cada índice. Esta mesma notação será bastante conveniente adiante.

A quantidade de coeficientes $d_{1}$ obtida depende da escolha dos índices, e será uma quantidade $p$ assim obtida:

$$
p=\left(\left|j_{1 M}-j_{1 m}\right|+1\right) \times \cdots \times\left(\left|k_{2 M}-k_{2 m}\right|+1\right)
$$

Assim sendo, no exemplo dado anteriormente, $p=1960$ coeficientes.

O ponto chave do trabalho é a obtenção dos coeficientes $d_{1}$; deste modo, a formação das matrizes $\mu$ e $f$ é de importância crucial, pois elas constituirão a base para o processamento numérico da equação (8).

A matriz $f$ é na realidade um vetor coluna que contém as $v\left(x_{i}, z_{j}\right)$, velocidades sísmicas nos pontos $\left(x_{i}, y_{j}\right)$ do modelo, obtidas numa malha de $n$ (eixo horizontal) por $m$ (eixo vertical) pontos: 


$$
\underline{f}=\left[\begin{array}{c}
v\left(x_{1}, y_{1}\right) \\
v\left(x_{1}, y_{2}\right) \\
\vdots \\
v\left(x_{1}, y_{m}\right) \\
v\left(x_{2}, y_{1}\right) \\
\vdots \\
v\left(x_{n}, y_{m}\right)
\end{array}\right]
$$

Já a matriz $\mu$ tem uma formatação um pouco mais complexa, conforme segue, sobretudo devido ao seu grande tamanho: $(n \cdot m)$ linhas $\times p$ colunas.

$\underline{\mu}=$

$$
\left[\begin{array}{c}
\psi_{j_{1 m}, k_{1 m}}\left(x_{1}\right) \cdot \psi_{j_{2 m}, k_{2 m}}\left(y_{1}\right) \cdots \psi_{j_{1 M}, k_{1 M}}\left(x_{1}\right) \cdot \psi_{j_{2 M}, k_{2 M}}\left(y_{1}\right) \\
\psi_{j_{1 m}, k_{1 m}}\left(x_{1}\right) \cdot \psi_{j_{2 m}, k_{2 m}}\left(y_{2}\right) \cdots \psi_{j_{1 M}, k_{1 M}}\left(x_{1}\right) \cdot \psi_{j_{2 M}, k_{2 M}}\left(y_{2}\right) \\
\vdots \\
\psi_{j_{1 m}, k_{1 m}}\left(x_{1}\right) \cdot \psi_{j_{2 m}, k_{2 m}}\left(y_{m}\right) \cdots \psi_{j_{1 M}, k_{1 M}}\left(x_{1}\right) \cdot \psi_{j_{2 M}, k_{2 M}}\left(y_{m}\right) \\
\psi_{j_{1 m}, k_{1 m}}\left(x_{2}\right) \cdot \psi_{j_{2 m}, k_{2 m}}\left(y_{1}\right) \cdots \psi_{j_{1 M}, k_{1 M}}\left(x_{2}\right) \cdot \psi_{j_{2 M}, k_{2 M}}\left(y_{1}\right) \\
\vdots \\
\psi_{j_{1 m}, k_{1 m}}\left(x_{n}\right) \cdot \psi_{j_{2 m}, k_{2 m}}\left(y_{m}\right) \cdots \psi_{j_{1 M}, k_{1 M}}\left(x_{n}\right) \cdot \psi_{j_{2 M}, k_{2 M}}\left(y_{m}\right)
\end{array}\right]
$$

A forma como os índices $j_{1}, j_{2}, k_{1}, k_{2}$ variam dos valores mínimos aos máximos na matriz dada pela equação (13) também é importante. Isto porque cada coeficiente $d_{1}$ obtido pela equação (8) é indexado e deve ser multiplicado por cada termo $\mu_{\mathbf{I}}(x, z)$ de mesmo índice na equação (9). A escolha aqui foi variar os índices na seguinte sequencia:

1. $k_{2}$ de $k_{2 m}$ à $k_{2 M}$;

2. $j_{2}$ de $j_{2 m}$ à $j_{2 M}$;

3. $k_{1}$ de $k_{1 m}$ à $k_{1 M}$;

4. $j_{1}$ de $j_{1 m}$ à $j_{1 M}$.

\section{Resultados}

\section{Modelo da falha dinstensional}

O primeiro modelo $\left(D I S_{1}\right)$ a ser tratado neste trabalho foi o da falha distensional, representando 10 camadas sedimentares (nem todas planas) em ambiente distensional. $O$ modelo foi suposto com $9 \mathrm{~km}$ de extensão e $3 \mathrm{~km}$ de profundidade. A propriedade geofísica de interesse é a velocidade das ondas sísmicas compressionais em subsuperfície. A representação do modelo está na figura 1 :

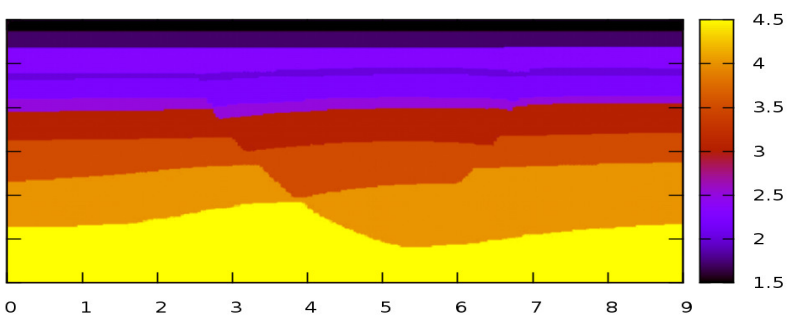

Figure 1: Modelo da falha distensional $D I S_{1}$.

\section{Parametrizações}

A primeira parametrização foi obtida utilizando-se 360 coeficientes; os índices foram $j_{1}=[0,2], j_{2}=[0,2], k_{1}=$ $[0,9], k_{2}=[0,3]$. O campo de velocidades obtido com esta parametrização encontra-se na figura 2 :

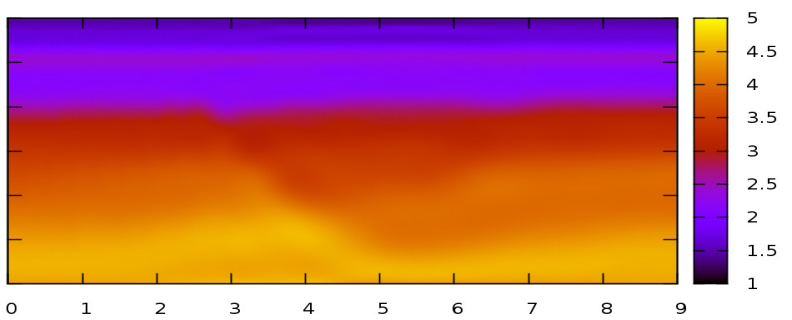

Figure 2: Parametrização do modelo da falha distensional $\left(P(D I S)_{1}\right)$ com ondaleta bidimensional chapéu mexicano, 360 coeficientes.

Já a segunda parametrização originou melhores resultados que a primeira, utilizando quase 0 dobro de coeficientes: 640. Os índices foram $j_{1}=[0,3]$, $j_{2}=[0,3], k_{1}=[0,9], k_{2}=[0,3]$. O campo de velocidades obtido com esta parametrização encontra-se na figura 2 :

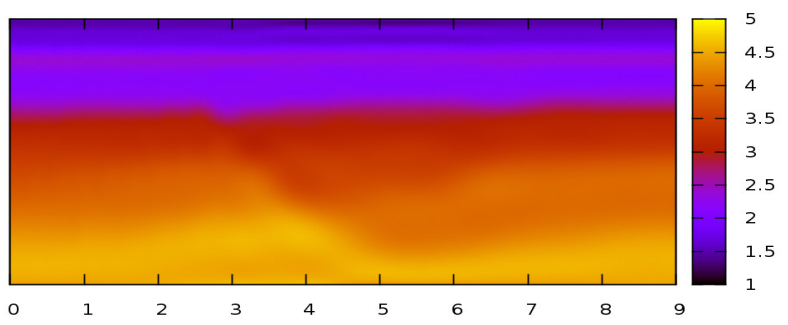

Figure 3: Parametrização do modelo da falha distensional $\left(P(D I S)_{2}\right)$ com ondaleta bidimensional chapéu mexicano, 640 coeficientes.

\section{Modelo Marmousi}

O modelo marmousi $\left(M A R_{1}\right)$ é conhecido pelo seu alto grau de complexidade. É composto por diversas camadas sedimentares, falhas, dobras e intrusões de altas velocidades; na figura 4 estão representadas as velocidade das ondas compressionais para um modelo também de $9 \mathrm{~km}$ de extensão e $3 \mathrm{~km}$ de profundidade:

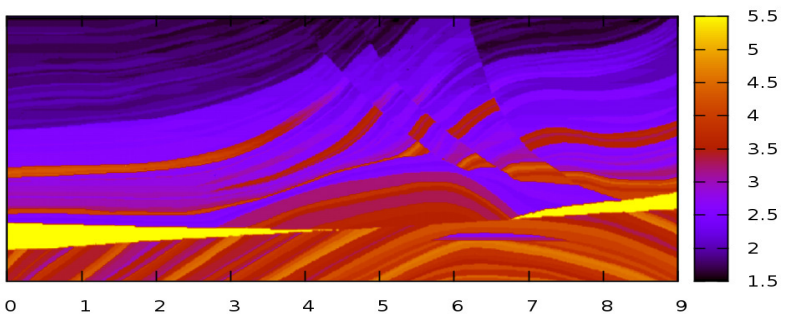

Figure 4: Modelo Marmousi $M A R_{1}$.

\section{Parametrizações}

Para o modelo marmousi, $M A R_{1}$, também foram tentadas parametrizações com os mesmos índices usados para 0 
modelo da falha distensional $D I S_{1}$ ou seja, primeiro com 360 coeficientes $\left(j_{1}=[0,2], j_{2}=[0,2], k_{1}=[0,9], k_{2}=\right.$ $[0,3])$ cujo resultato aparece na figura 5 , e depois com 640 coeficientes $\left(j_{1}=[0,3], j_{2}=[0,3], k_{1}=[0,9], k_{2}=[0,3]\right)$, conforme figura 6 .

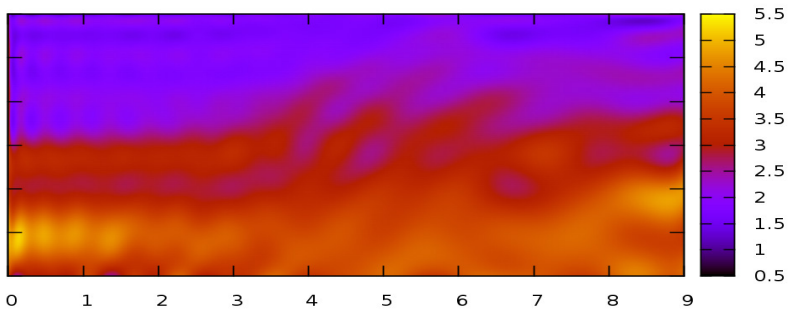

Figure 5: Parametrização do modelo Marmousi $\left(P(M A R)_{1}\right)$ com ondaleta bidimensional chapéu mexicano, 360 coeficientes.

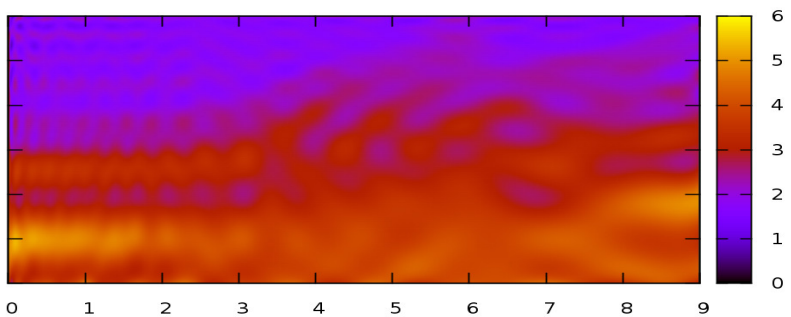

Figure 6: Parametrização do modelo Marmousi $\left(P(M A R)_{2}\right)$ com ondaleta bidimensional chapéu mexicano, 6400 coeficientes.

\section{Conclusões}

A parametrização de campos de velocidaes sísmicas utizando a ferramenta das ondaletas bidimensionais, parece indicar um caminho promissor. Apesar da ainda grande quantidade de coeficientes, os resultados obtidos são muito bons para a o modelo da falha distensional (tanto com 640, quanto com 360 coeficientes). A maior quantidade de coeficientes realçou detalhes, mas pode acarretar um aumento do custo computacional nos processos de modelagem e inversão.

A técnica gerou resultados razoáveis para o modelo marmousi (é possível perceber as principais feições, ainda que apareçam alguns artefatos). A maior quantidade de coeficientes, neste caso, realçou mais artefatos do que detalhes importantes, se comparado com o modelo original.

Não obstante, uma técnica de filtragem que elimine coeficiente nulos (e aqueles quase nulos), bem como que reúna em um só valor coeficientes muito próximos uns dos outros, pode ajudar a reduzir a quantidade de parâmetros.

\section{Agradecimentos}

Os autores agradecem: ao CPGG-UFBA e à ANP pelo oferecimento de condições para a realização deste trabalho.

\section{bibliografia}

Bastos, I.P. Figueiró, W.M.; 2013. Parametrização unidimensional de campos de velocidades sísmicas por ondaleta de Morlet. 13th International Congress of the Brazilian Geophysical Society, Expanded Abstracts, Rio de Janeiro.

Martinez, A.C. Figueiró, W.M.; 2011. Parametrização ondaleta do campo de velocidades sísmicas relativo a um domo salino. 12th International Congress of the Brazilian Geophysical Society, Expanded Abstracts, Rio de Janeiro.

Morettin, P.A.; 1999. Ondas e Ondaletas: da Análise de Fourier à Análise de Ondaletas. EDUSP, São Paulo.

Perin, H.M. Figueiró, W.M.; 2011. Parametrização de campo bidimensional de velocidades sísmicas usando ondaletas. 12th International Congress of the Brazilian Geophysical Society, Expanded Abstracts, Rio de Janeiro. 